\title{
Testing nested and non-nested periodically integrated autoregressive models
}

\author{
Philip Hans Franses \\ Econometric Institute \\ Erasmus University Rotterdam \\ P.O.Box 1738 \\ 3000 DR Rotterdam \\ The Netherlands \\ Michael McAleer \\ Department of Economics \\ University of Western Australia \\ Nedlands, Perth WA 6009 \\ Australia
}

December 18, 1996

Keywords: seasonality, model selection, periodicity.

\begin{abstract}
Periodically integrated time series require a periodic differencing filter to remove the stochastic trend. A non-periodic integrated time series needs the first-difference filter for similar reasons. When the changing seasonal fluctuations for the non-periodic integrated series can be described by seasonal dummy variables for which the corresponding parameters are not constant within the sample, such a series may not be easily distinguished from a periodically integrated time series. In this paper, nested and non-nested testing procedures are proposed to distinguish between these two alternative stochastic and non-stochastic seasonal processes,
\end{abstract}


when it is assumed there is a single unknown structural break in the seasonal dummy parameters. Several empirical examples using quarterly real macroeconomic time series for the United Kingdom illustrate the nested and non-nested approaches.

\section{Introduction}

Many quarterly observed macroeconomic time series have the following three properties: (i) after trend removal, seasonal variation is the dominant source of variation, (ii) seasonal variation is not constant over time, and (iii) seasonality is often related to the trend and the stage of the business cycle, see Hylleberg (1994) and Franses (1996a) inter alia. In Franses (1996b) it is shown that the class of the periodically integrated autoregressive [PIAR] time series model allows the joint description of these three features, which goes beyond the capabilities of the standard seasonal ARIMA model (see, e.g., Prothero and Wallis, 1976) and the structural model (see, Harvey and Todd, 1983). A key property of PIAR time series is that they need a seasonally varying differencing filter to remove the stochastic trend. Given the results in, e.g., Perron (1989), it is conceivable that this seasonal variation in the differencing filter is due to neglecting seasonal mean shifts in a periodic model for an otherwise regular first difference series. In fact, such mean shifts may mimic seasonal diverging trends, which in turn are then picked up by the PIAR model.

In this paper, attention is focused on the selection between such an integrated process with non-constant intercept parameters and a PI process. Since these two processes may be interpreted as non-nested models, non-nested testing procedures may be appropriate. Furthermore, given that a PI model with shifts in the intercept parameters may also be regarded as a reasonable description of the data, a nested testing procedure is also considered. It is assumed, without loss of generality, that there is a single unknown one-time parameter change for the seasonal dummy variables. A simple method to test for the presence of such a change is also presented.

The outline of the paper is as follows. In section 2, the research question is motivated by highlighting specific aspects of the two models. Nested and non-nested testing procedures are proposed in section 3 , and both procedures are illustrated for six quarterly real macroeconomic time series for the United Kingdom in section 4 . One of the main findings is that the PIAR model is often improved by allowing for seasonal mean shifts. Some concluding remarks are given in section 5 .

\section{Motivation}

\subsection{Periodic integration}

Consider a macroeconomic time series $y_{t}$ which is observed at quarterly intervals over $N$ years, where $t$ is the quarterly index running from $1, \ldots, n$, and $n=4 N$. A periodic autoregressive process of order $p, \operatorname{PAR}(p)$, can be represented by 


$$
y_{t}=\mu_{s}+\phi_{1 s} y_{t-1}+\cdots+\phi_{p s} y_{t-p}+\epsilon_{t}
$$

where the index $s$ denotes the parameter values that can vary over the four seasons, $s=1, \ldots, S$, where $S$ is set equal to 4 . The expression in (1), and all subsequent representations, can also be useful when $S$ takes such values as 2, 6 or 12 , but in this paper the analysis concentrates on quarterly series. The $\epsilon_{t}$ are assumed to follow a standard white noise process. In some applications, it may be useful to replace $\epsilon_{t}$ by $\epsilon_{s t}$, but this extension does not affect the analysis except for the application of a straightforward modified estimation method to allow for seasonal heteroskedasticity. Concise discussions of estimation aspects of periodic time series models can be found in, e.g., Pagano (1978) and Vecchia and Ballerini (1991), inter alia. The parameters in model (1) can be estimated by applying ordinary least squares to

$$
y_{t}=\sum_{s=1}^{S} D_{s t} \mu_{s}+\sum_{s=1}^{S} D_{s t}\left(\phi_{1 s} y_{t-1}+\cdots+\phi_{p s} y_{t-p}\right)+\epsilon_{t}
$$

where the $D_{s t}$ are seasonal dummy variables which equal one in season $s$ and zero in all other seasons, and the number of parameters is $(p+1) S$.

For the purpose of investigating stochastic trend properties of $y_{t}$, a more convenient representation of (1) relies on stacking the $y_{t}$ observations in the $(4 \times 1)$ vector $Y_{T}=\left(Y_{1, T}, \ldots, Y_{4, T}\right)^{\prime}$, where $T$ runs from $1, \ldots, N, N=n / 4$ and $Y_{s, T}$ is the observation in season $s$ in year $T$ (see, e.g., Osborn (1991), Franses (1994), and Tiao and Grupe (1980)). Note that equation (1) is already a nonstationary process in the sense that the autocorrelation function varies with the season. Using the stacked observation notation, model (1) can be rewritten as

$$
A_{0} Y_{T}=\mu+A_{1} Y_{T-1}+\ldots+A_{m} Y_{T-m}+\epsilon_{T}
$$

where the $A_{j}, j=0, \ldots, m$, are $(4 \times 4)$ parameter matrices, with $A_{0}$ being lower triangular with ones on the diagonal, and the $\mu$ and $\epsilon_{T}$ containing the stacked $\mu_{s}$ and $\epsilon_{t}$. The value of $m$ depends on $p$ in (1) according to $m \leq[1+(p-1) / 4]$. To check for unit roots in the $\operatorname{PAR}(p)$ process, it is necessary to investigate whether the solutions to

$$
\left|A_{0}-A_{1} z-\cdots-A_{m} z^{m}\right|=0
$$

are on or outside the unit circle. Boswijk and Franses (1996) propose a test for the presence of at most a single unit root in a PAR $(p)$ process, while Boswijk, Franses and Haldrup (1996) extend their method to multiple unit roots. Franses and Paap (1994) find empirical support for the presence of only a single unit root in a large number of illustrative series for the UK economy.

In the case of a single unit root, the three cointegrating relations among the $Y_{s, T}$ variables are $Y_{4, T}-\alpha_{4} Y_{3, T}, Y_{3, T}-\alpha_{3} Y_{2, T}$ and $Y_{2, T}-\alpha_{2} Y_{1, T}$. Since $Y_{4, T}-$ $Y_{4, T-1}$ is a stationary variable, it follows that $Y_{1, T}-\alpha_{1} Y_{4, T-1}$ is a stationary process, where $\alpha_{1}=1 / \alpha_{2} \alpha_{3} \alpha_{4}$. This implies that in the case of a single unit root the model in (1) can be rewritten as

$$
\left(1-\alpha_{s} B\right) y_{t}=\mu_{s}+\beta_{1 s}\left(1-\alpha_{s-1} B\right) y_{t-1}+\cdots+\beta_{p-1, s}\left(1-\alpha_{s-p+1} B\right) y_{t-p+1}+\epsilon_{t}
$$


subject to the non-linear restriction

$$
\alpha_{1} \alpha_{2} \alpha_{3} \alpha_{4}=1
$$

where $B$ is the familiar backward shift operator. Note that $\alpha_{-k}=\alpha_{4-k}$ for $k=0,1,2 \ldots$ The parameters $\alpha_{s}$ and $\beta_{j}$ are functions of the parameters $\phi_{p s}$ in (1). The $\left(1-\alpha_{s} B\right)$ filter is called the periodic differencing filter. When $y_{t}$ can be described as (4) with (5), while $\alpha_{s} \neq 1$ for all $s$, the $y_{t}$ series is said to be periodically integrated [PIAR]. The model in (4) subject to (5) can be estimated using non-linear least squares [NLS]. Since $(1-\hat{\alpha} B) y_{t}$ is a periodically stationary time series, where $\hat{\alpha}_{s}$ is the non-linear least squares estimator of $\alpha_{s}$, the $t$ ratios for the estimated $\beta_{i \text { s }}$ parameters in (4) can be compared with the fractiles of the asymptotic standard normal distribution, see Franses (1996b).

\subsection{Regular differencing and seasonal mean shifts}

Model (4)-(5) with $\mu_{s} \neq 0$ implies that $Y_{s, T}-\alpha_{s} Y_{s-1, T}-\delta_{s}(s=1,2,3,4)$ are stationary time series. When $\delta_{s} \neq 0$ and $\alpha_{s} \neq 1$, this implies that $Y_{s, T}$ and $Y_{s-1, T}$ may have diverging or converging trends, depending on the values of $\alpha_{s}$ and $\delta_{s}$. Hence it is clear that the PIAR model allows for changing seasonality, whereby seasons can even switch. The latter feature, however, may be caused by changing seasonal means instead of by seasonally varying $\alpha_{s}$ values. In other words, it may be that $Y_{s, T}-Y_{s-1, T}-\delta_{s}-I_{(t \geq \tau)} \delta_{s}^{*}$ are stationary time series instead, where $I_{(t \geq \tau)}$ is an indicator function which is equal to 1 when $t \geq \tau$. Notice that $\alpha_{s}=1$ in (4) also amounts to a single unit root in the $Y_{T}$ vector series, implying that the latter set of stationary variables also amounts to only a single stochastic trend in $y_{t}$.

It seems therefore reasonable to contrast the PIAR model in (4)-(5) with a model that considers the first-order differenced time series, $(1-B) y_{t}$, when it can be described using a (periodic) autoregression and allows for a one-time structural break at given time $\tau$, namely

$$
(1-B) y_{t}=\delta_{s}+I_{(t \geq \tau)} \delta_{s}^{*}+\beta_{1 s}(1-B) y_{t-1}+\cdots+\beta_{p-1, s}(1-B) y_{t-p+1}+\epsilon_{t} .
$$

In this case, $y_{t}$ is a periodic autoregressive integrated time series [PARI]. Notice that (4)-(5) and (6) can give quite different out-of-sample forecasts, particularly for longer horizons. In (6), the number of parameters is $8+4(p-1)$. For convenience, it is assumed that the model remains periodic so that the $\beta$ parameters vary seasonally. The $\delta_{s}^{*}$ parameters have values that may be different from zero, which implies that the growth rates can change at time $\tau$. Of course, before testing (6) against (4)-(5), it should be verified whether the model in (6) is statistically adequate in terms of uncorrelated and homoskedastic errors and the absence of non-normality. In the next section, testing procedures are proposed for choosing between (4)-(5) and (6) when they are both nested within a more general model and also when they are non-nested. 


\section{Nested and non-nested testing procedures}

\subsection{Nested Procedures}

If the time series models given in (4)-(5) and (6) are essentially based on empirical observation, then the theoretical foundations of the competing models may not be of great substance. In such a context, when the two models are simply competing empirical explanations which do not preclude a more general explanation, a straightforward method of testing each of these models is to nest both in the more general model, with $3+8+4(p-1)$ parameters, given by

$$
\begin{aligned}
\left(1-\alpha_{s} B\right) y_{t} & =\beta_{1 s}\left(1-\alpha_{s-1} B\right) y_{t-1}+\cdots+\beta_{p-1, s}\left(1-\alpha_{s-p+1} B\right) y_{t-p+1} \\
& +\kappa_{s}+I_{(t \geq \tau)} \kappa_{s}^{*}+\epsilon_{t}
\end{aligned}
$$

which is to be estimated subject to (5). It is assumed, in the first instance, that $\tau$ is known. Equation (7) is not only a convenient and obvious method of testing both (4)-(5) and (6), but is also useful in that it might be an empirically acceptable model. The validity of (7) rests in its ability to accommodate both periodic integration and a structural break. It should be noted that the structural break is accommodated within the periodically integrated model, so that the non-linear restriction is imposed in estimating (7). The general model (7), in which all variables are (periodically) stationary, may be estimated using NLS, which yields a residual sum of squares $R S S_{G}$.

The PI model in (4)-(5) implies that there is no structural break, so that the $\kappa_{s}^{*}$ parameters in (7) are equal to zero. Since the $\kappa_{s}^{*}$ are coefficients of stationary variables, the residual sum of squares of the PI model, say $R S S_{P I}$, can be compared with $R S S_{G}$ from (7) using the standard $F$ test. This test will be denoted $F_{P I}(4, n-k)$, where $k$ is the number of parameters in the general model and PI in (4)-(5) is the null hypothesis.

The PARI time series model (6) implies that each of the $\alpha_{s}$ in (7) is equal to one, in which case the non-linear restriction is satisfied automatically. Hence, (6) also assumes there is a single unit root in the multivariate representation (2). Comparing these models does not involve a change in the number of unit roots. When each $\alpha_{s}$ is equal to one, the $\kappa_{s}$ and $\kappa_{s}^{*}$ in (7) equal $\delta_{s}$ and $\delta_{s}^{*}$, respectively. Denoting the residual sum of squares from estimating (6) by OLS as $R S S_{I}$, model (6) can be tested against ( 7$)$ using the standard $F$ test, denoted as $F_{I}(3, n-k)$, where $k=4 p+7$ in $(7)$ and $I$ is the null hypothesis. The three restrictions to be tested arise from the fact that (7) is estimated under the non-linear restriction (5), in which case there are only three free $\alpha_{s}$ parameters.

A further restriction to be tested on (7) is that each of the $\alpha_{s}$ is equal to one and that the $\kappa_{s}^{*}$ are equal to zero, wherein the integrated model with no structural break is the null hypothesis. Such a test is different from testing each of the $\delta_{s}^{*}$ in (6) to be equal to zero, since (6) has already imposed the restriction that the $\alpha_{s}$ are equal to unity. Consequently, the powers of the tests are likely to differ, depending on which of $(6)$ or $(7)$ is correct. In the context of nested 
tests, (7) is the maintained hypothesis, whereas (4)-(5), (6), and (6) with the $\delta_{s}^{*}$ set to zero are the respective null hypotheses.

The interpretation to be given to these test statistics is straightforward. A significantly large value of $F_{P I}\left(F_{I}\right)$ leads to the rejection of the PI (I) model in (4)-(5) ((6)) in favour of the general model with both periodic integration and a structural break. A significant test statistic for the null hypothesis of an integrated model with no structural break also leads to the general model (7). Insignificant test statistics in each of these three cases leads to non-rejection of the respective null hypotheses. It should be emphasized that, in the nested case, the general model given in (7) may not only be statistically valid but may also be interpreted as a useful model in itself.

\subsection{Non-nested procedures}

When the models in (4)-(5) and (6) are non-nested, in that using the standard $F$ test in a model such as (7) cannot be entertained, non-nested testing procedures should be used. Dastoor and McAleer (1989) propose four classifications of tests for competing non-nested linear regression models, depending on whether there are exactly two or more than two competing linear regression models. For linear regression models in the absence of linear or non-linear parametric restrictions, several procedures are available. In the case of two non-nested models considered here, a distinction is made between the Cox-type tests of Pesaran (1974), Davidson and MacKinnon (1981) and Fisher and McAleer (1981), and the nonnested $F$ tests of Deaton (1982), Dastoor (1983), Gourieroux et al. (1983), Mizon and Richard (1986) (based on encompassing), and McAleer and Pesaran (1986) (based on Roy's (1953) union-intersection principle). It should be noted that the nested $F$ test given in (7) is not a non-nested test of (4)-(5) versus (6) based on the non-nested procedures just mentioned. Moreover, Monte Carlo results indicate that non-nested tests are generally more powerful than nested $F$ tests based on a general model such as (7).

Pesaran and Hall (1988) derive Cox-type tests of two non-nested linear regression models subject to linear restrictions, and incorporate small sample adjustments for the mean and variance of the statistic under the assumption of non-stochastic regressors. If the models are subject to non-homogeneous linear restrictions, substituting the restrictions yields transformed dependent variables which differ between the two non-nested models. In testing (4)-(5) and (6) against each other, it is possible to obtain several asymptotically equivalent Cox-type tests, but small sample adjustments are not appropriate given the nature of the regressors. For present purposes, the most computationally convenient method of testing the two models is to use weights $(1-\lambda)$ and $\lambda$ for the models given in (4) and (6), respectively, to yield

$$
\begin{aligned}
& (1-\lambda)\left(1-\alpha_{s} B\right) y_{t}+\lambda(1-B) y_{t}= \\
& (1-\lambda)\left[\mu_{s}+\beta_{1 s}\left(1-\alpha_{s-1} B\right) y_{t-1}+\cdots+\beta_{p-1, s}\left(1-\alpha_{s-p+1} B\right) y_{t-p+1}\right]+ \\
& \lambda\left[\delta_{s}+I_{(t \geq r)} \delta_{s}^{*}+\beta_{1 s}(1-B) y_{t-1}+\cdots+\beta_{p-1, s}(1-B) y_{t-p+1}\right]+\epsilon_{t}
\end{aligned}
$$


which is to be estimated subject to (5). In (8), $\lambda$ is not identifiable without imposing restrictions. Since there are several methods for identifying $\lambda$ (see McAleer and Pesaran (1986) for further details), the method adopted here is to use sample information. Where such information is obtained from the alternative non-nested model, the outcome is a $J$-type test (see Davidson and MacKinnon (1981)). Specifically, to test $H_{0}: \lambda=0$ in (8), estimates of the parameters from (6) are used to replace the unknown parameters as follows:

$$
\begin{aligned}
& (1-\lambda)\left(1-\alpha_{s} B\right) y_{t}+\lambda(1-B) y_{t}= \\
& (1-\lambda)\left[\mu_{s}+\beta_{1 s}\left(1-\alpha_{s-1} B\right) y_{t-1}+\cdots+\beta_{p-1, s}\left(1-\alpha_{s-p+1} B\right) y_{t-p+1}\right]+ \\
& \lambda\left[\hat{\delta}_{s}^{*}+I_{(t \geq \tau)} \hat{\delta}_{s}^{*}+\hat{\beta}_{1 s}(1-B) y_{t-1}+\cdots+\hat{\beta}_{p-1, s}(1-B) y_{t-p+1}\right]+\epsilon_{t}
\end{aligned}
$$

which is to be estimated subject to (5). See Davidson and MacKinnon (1981) for a justification of this procedure. Since all the variables in (9) are (periodically) stationary, NLS applied to (9)-(5) yields a $t$ ratio for the estimate of $\lambda$ which is asymptotically distributed as standard normal. Let the $t$ ratio for the test of PI in (4)-(5) against $I$ in (6) be denoted as $t_{P I}$.

A test of $H_{1}: \lambda=1$ in (8), namely testing (6) against (4)-(5), may be obtained by replacing the unknown parameters of (4) with estimates from (4)(5) as follows:

$$
\begin{aligned}
& (1-\lambda)\left(1-\hat{\alpha}_{s} B\right) y_{t}+\lambda(1-B) y_{t}= \\
& (1-\lambda)\left[\hat{\mu}_{s}+\beta_{1 s}\left(1-\hat{\alpha}_{s-1} B\right) y_{t-1}+\cdots+\hat{\beta}_{p-1, s}\left(1-\hat{\alpha}_{s-p+1} B\right) y_{t-p+1}\right]+ \\
& \quad \lambda\left[\delta_{s}+I_{(t \geq \tau)} \delta_{s}^{*}+\beta_{1 s}(1-B) y_{t-1}+\cdots+\beta_{p-1, s}(1-B) y_{t-p+1}\right]+\epsilon_{t}
\end{aligned}
$$

which is not estimated subject to (5). Since all the variables in (10) are (periodically) stationary, NLS applied to (10) yields a $t$ ratio for the estimate of $(1-\lambda)$ which is asymptotically distributed as standard normal. Let the $t$ ratio for the test of $I$ in (6) against PI in (4)-(5) be denoted as $t_{I}$.

There are four possible outcomes for the non-nested testing procedure. First, $t_{P I}$ is significant while $t_{I}$ is not, so PI is rejected while $\mathrm{I}$ is not. Hence, the integrated time series model $(6)$ is selected. Second, $t_{I}$ is significant while $t_{P I}$ is not, in which case the PI model is selected over the I model. Third, neither $t_{P I}$ nor $t_{I}$ is significant, so the tests may lack power because neither model is rejected. Consequently, both the PI and I models may be adequate representations of the data. Fourth, both $t_{P I}$ and $t_{I}$ are significant, which implies that neither of the models (4)-(5) nor (6) adequately describes the series, even though misspecification may not have been detected for either of the models using standard diagnostic checks. This outcome indicates that each of the models should be modified in some way.

It should be noted that equations (8), (9) and (10) are not models for interpretation, but are simply devices for obtaining test statistics for the two non-nested models. Therefore, neither (9) nor (10) has an interpretive value, unlike the more general model (7) in the nested case.

In the theoretical derivation of the tests above, it has been presumed that the value of $\tau$ is known. When $\tau$ is not known exactly, an important practical 
question which would arise is the determination of the value of $\tau$ empirically. Suppose that a PI model has been estimated and it is then desired to check whether (6) is more appropriate. The value of $\tau$ may be determined by applying parameter constancy tests to (4)-(5). Such a procedure necessarily involves a series of tests, which will affect the overall significance level of the tests in an unknown way. An alternative proposal is to use the likelihood ratio (LR) test statistic

$$
L R=n \log \left(R S S /\left(R S S_{1}+R S S_{2}\right)\right)
$$

for each subsample defined by various choices for $\tau$, where RSS is the residual sum of squares that corresponds to the entire sample, while $R S S_{1}$ and $R S S_{2}$ correspond to the first and second subsamples. This $L R$ statistic concerns a test for coefficient stability conditional on variance equality (see Pesaran et al. (1985) for an overview of parameter constancy tests). In the case of an unknown change-point, it is possible to use the test statistic $\sup (L R)$, which is the largest LR value over all possible $\tau$. The distribution of $\sup (L R)$ is derived in Andrews (1993) for a wide class of models, including non-linear models. However, it must be recognized that determining the value of $\tau$ endogenously has a non-vanishing effect on the asymptotic distribution of the proposed tests.

\section{Empirical applications}

In this section the nested and non-nested testing procedures developed above are applied to six empirical applications. These applications concern the logarithms of quarterly real macroeconomic time series for the United Kingdom over the effective sample period 1956.1-1988.4. The variables are GDP, Total Investment ("Investment"), Exports, Imports, Total Consumption ("Consumption") and Consumption of Nondurables ("Nondurables"). In Franses and Paap (1994), these variables are found to be periodically integrated of order one, see also Franses (1996b). The variables GDP, Investment and Exports can be described using PIAR(2) models, while the other three variables can be described using PIAR(1) models. To obtain an impression of typical $\alpha_{s}$ values, their estimates are reported in Table I. It is evident that these values are very close to unity. The standard errors are usually smaller than 0.01 , although these should be treated with care since the time series contain a stochastic trend. Formal tests for the adequacy of the $(1-B)$ filter are performed in Franses and Paap (1994), and the overall result is that this restriction on the $\alpha_{s}$ parameters can be rejected, with probability values typically smaller than 0.01 .

The proposed tests require that $\tau$ be known exactly. A common value of $\tau$ would be to select the break-point at $\tau=1969.1$, corresponding to the year following a major VAT increase in the UK. However, to test for a structural break at an unknown change-point in each of the six PIAR models, the $L R$ test statistic (11) was calculated for various values of $\tau$. Since it is desired to estimate each parameter with the same number of observations, $\tau$ was always set equal to the first quarter of each year. The $L R$ test is calculated for $\tau$ ranging from 1960.1 through to 1984.1, which ensures that there are at least 20 observations in the subsamples to estimate the parameters. The largest value of the $L R$ statistics, 
Table I

Periodic Differencing Filter $\left(1-\hat{\alpha}_{s} B\right)$ based on PIAR $(p)$ Models for Six UK Macroeconomic Time Series over 1956.1-1988.4

\begin{tabular}{lcllll}
\hline \hline Variable & Order of PIAR & $\hat{\alpha}_{1}$ & $\hat{\alpha}_{2}$ & $\hat{\alpha}_{3}$ & $\hat{\alpha}_{4}$ \\
\hline GDP & 2 & 1.007 & 0.916 & 1.069 & 1.014 \\
Investment & 2 & 1.050 & 0.885 & 1.074 & 1.003 \\
Exports & 2 & 0.947 & 1.025 & 1.036 & 0.994 \\
Imports & 1 & 0.965 & 1.028 & 0.983 & 1.025 \\
Consumption & 1 & 1.036 & 0.912 & 1.058 & 1.000 \\
Nondurables & 1 & 1.002 & 0.929 & 1.033 & 1.039 \\
\hline \hline
\end{tabular}

Note: The periodic differencing filter is estimated under the non-linear restriction $\alpha_{4}=1 /\left(\alpha_{1} \alpha_{2} \alpha_{3}\right)$. The statistical package MicroTSP (version 7.0) is used for these computations. The PIAR models pass diagnostic checks for first and first-to-fourth order residual autocorrelation, first order periodic autocorrelation and first order ARCH.

denoted $\sup (L R)$, is reported in Table II, and the corresponding value of $\tau$ is given in the next column. The final column of Table II contains the $5 \%$ critical values for $\sup (L R)$, which have been interpolated from the asymptotic critical values displayed in Table I in Andrews (1993).

In Table III are reported the results of the nested and non-nested test procedures for the six UK variables. The third and fourth columns of Table III provide the nested test statistics. It is concluded from these results that the PI model with breaks, i.e. the nesting model, is preferred over the nested PI and I models for five of the six variables, since both the PI and I models are rejected. For Exports, the PI model is preferred since the I model is rejected but the PI model is not. Except for Exports and Nondurables, where the PI model is preferred and both models are rejected, respectively, the non-nested test results in columns five and six of Table III give different results from those using the nested testing approach. It should be emphasized that, in contrast to the rejection of both the PI and I models in favour of the general model for Nondurables in the nested case, rejection of PI and I using non-nested tests does not suggest whether an appropriate model exists. For Investment, the statistics do not reject either the PI model or the I model. For Imports and GDP, the PI specification is rejected in favour of the I model with a structural break, while for Consumption it is the reverse.

Since the nested and non-nested test procedures do not generally lead to agreement, the values of the Schwarz model selection criterion for the models (4)-(5), (6) and (7) are also calculated, and are reported in Table IV. From 
Table II

Testing for a Structural Break in PIAR Models

\begin{tabular}{lclll}
\hline \hline Variable & Order of PIAR & $\sup (L R)^{(1)}$ & $\tau^{(2)}$ & $5 \%$ level $^{(3)}$ \\
\hline GDP & 2 & $42.718^{*}$ & 969.1 & 25.2 \\
Investment & 2 & $34.855^{*}$ & 1964.1 & 27.6 \\
Exports & 2 & $29.585^{*}$ & 1979.1 & 27.0 \\
Imports & 1 & 16.001 & 1967.1 & 19.4 \\
Consumption & 1 & 16.906 & 1969.1 & 18.8 \\
Nondurables & 1 & $35.815^{*}$ & 1969.1 & 18.8 \\
\hline \hline
\end{tabular}

* Significant at the $5 \%$ level.

Notes

(1) The Likelihood Ratio test, $L R$, refers to a test for coefficient stability conditional on variance stability in PIAR models, under the non-linear restriction $\alpha_{1} \alpha_{2} \alpha_{3} \alpha_{4}=1$. Sup $(L R)$ refers to the largest value of the $L R$ test values for all break-points considered. The $L R$ test corresponds to the familiar analysis of covariance Chow test (see Pesaran et al. (1985)).

(2) This value of $\tau$ is the first quarter of the second sample period and is used to calculate the $L R$ test statistics. The $L R$ test compares the residual sum of squares of the PIAR model for 1956.1- $(\tau-1)$ with that of $\tau-1988.4$.

(3) Critical values at the $5 \%$ level for the $\sup (L R)$ test in case of an unknown break-point. These values are interpolated from the asymptotic critical values displayed in Table I in Andrews (1993). There are 7 parameters to be estimated in the PIAR(1) model and 11 in the PIAR(2) model. The fraction of the sample 1956.1-1988.4 for which there may be a break-point (denoted as $\pi_{0}$ in Andrew's Table I) can be calculated using the value of $\tau$.

Table III

Nested and Non-nested Test Statistics for Six UK Macroeconomic Time Series

\begin{tabular}{lcllll}
\hline \hline Variable & Break-point & \multicolumn{2}{c}{ Nested tests ${ }^{(1)}$} & \multicolumn{2}{c}{ Non-nested tests } \\
& $\tau$ & $F_{P I}$ & $F_{I}$ & $t_{P I}$ & $t_{I}$ \\
\hline GDP & 1969.1 & $6.152^{*}$ & $6.394^{*}$ & $3.083^{*}$ & 1.738 \\
Investment & 1964.1 & $3.233^{*}$ & $4.161^{*}$ & 1.939 & 1.891 \\
Exports & 1979.1 & 1.317 & $3.256^{*}$ & 1.423 & $2.789^{*}$ \\
Imports & 1967.1 & $3.194^{*}$ & $3.999^{*}$ & $2.164^{*}$ & 1.827 \\
Consumption & 1969.1 & $3.252^{*}$ & $15.97^{*}$ & -0.033 & $6.083^{*}$ \\
Nondurables & 1969.1 & $4.871^{*}$ & $10.45^{*}$ & $2.213^{*}$ & $4.416^{*}$ \\
\hline \hline
\end{tabular}

* Significant at the $5 \%$ level.

Note:

${ }^{(1)}$ The $\mathrm{F}_{P I}$ test has $(4,117)$ and $(4,121)$ degrees of freedom for PIAR(2) and PIAR(1) models, respectively, while the $F_{I}$ test has $(3,117)$ and $(3,121)$ degrees of freedom, respectively. 
Table IV

Schwarz Criteria for Nested and Non-nested PIAR Models

\begin{tabular}{lclll}
\hline \hline & \multicolumn{3}{l}{ Model } \\
\hline Variable & $\begin{array}{c}\text { Break-point } \\
\tau\end{array}$ & PI & I with break & PI with break \\
\hline GDP & 1969.1 & -1029.9 & -1030.1 & $-1035.5^{* *}$ \\
Investment & 1964.1 & $-843.51^{* *}$ & -839.09 & -837.82 \\
Exports & 1979.1 & $-833.37^{* *}$ & -823.72 & -819.66 \\
Imports & 1967.1 & $-835.54^{* *}$ & -831.43 & -829.26 \\
Consumption & 1969.1 & $-1083.8^{* *}$ & -1048.4 & -1077.8 \\
Nondurables & 1969.1 & -1162.8 & -1147.2 & $-1163.0^{* *}$ \\
\hline \hline
\end{tabular}

Note:

* Smallest value of the Schwarz criterion, i.e. the corresponding model is selected.

these values, the PI model yields the most parsimonious description of the data for four of the six variables, while the PI model with a break in (7) is selected for GDP and Nondurables. The I model with a break in (6) is not chosen for any variable. Overall, it can be concluded that the various testing and model selection methods do not lead to a specific model being preferred. In general, it seems that the PI model with a structural break may be useful for GDP and Nondurables. The estimation results for the latter variable, by way of illustration, are

$$
\left(1-\alpha_{s} B\right) y_{t}=\delta_{s}+I_{(t \geq \tau)} \delta_{s}^{*}+\epsilon_{t}
$$

where $\tau$ is 1969.1

$$
\begin{array}{rrrr}
\hat{\alpha}_{1}=1.051 & \hat{\alpha}_{2}=0.929 & \hat{\alpha}_{3}=1.022 & \hat{\alpha}_{4}=1 /\left(\hat{\alpha}_{1} \hat{\alpha}_{2} \hat{\alpha}_{3}\right)=1.002 \\
(0.015) & (0.013) & (0.014) & (0.015)
\end{array}
$$

and

$$
\begin{array}{rrrr}
\hat{\delta}_{1}=-0.617 & \hat{\delta}_{2}=0.789 & \hat{\delta}_{3}=-0.214 & \hat{\delta}_{4}=0.018 \\
(0.151) & (0.137) & (0.148) & (0.144) \\
\hat{\delta}_{1}^{*}=-0.024 & \hat{\delta}_{2}^{*}=0.000 & \hat{\delta}_{3}^{*}=0.005 & \hat{\delta}_{4}^{*}=0.019 \\
(0.007) & 0.006) & (0.006) & (0.006)
\end{array}
$$

where the asymptotic standard errors are given in parentheses. It might be desired to simplify this model even further since not all $\delta_{s}$ and $\delta_{s}^{*}$ are statistically significant. It is clear, however, that $\hat{\delta}_{1}^{*}$ and $\hat{\delta}_{4}^{*}$ are significantly different from zero, and that the estimates of the $\alpha_{s}$ parameters are close, though not equal, to unity. 
For Investment, Exports and Consumption, it might be concluded that a PI model without a break is useful, although the evidence is not equally strong for all variables. For Imports, a model like (6) might be specified. The corresponding results, after deleting insignificant $\hat{\delta}_{s}^{*}$ estimates, are $\hat{\delta}_{1}=0.014(0.007), \hat{\delta}_{2}=$ $0.035(0.007), \hat{\delta}_{3}=0.032(0.009), \hat{\delta}_{4}=-0.037(0.009)$ and $\hat{\delta}_{34}^{*}=0.030(0.009)$, where $\hat{\delta}_{34}^{*}$ refers to the variable with -1 in the third quarter and +1 in the fourth quarter, starting in 1967. Hence, the total seasonal variation has not changed, since the seasonal component in quarter 3 has decreased by the same amount as the increase in the seasonal component in quarter 4 .

\section{Concluding remarks}

This paper has been concerned with testing alternative specifications of integrated time series. Two specifications were considered, namely one which is periodically integrated with periodic differencing filter given by $\left(1-\alpha_{s} B\right.$ ) (where not all $\alpha_{s}$ equal unity), and an integrated time series with filter $(1-B)$ which requires seasonal dummy variables with corresponding parameters subject to change over the sample. Simple nested and non-nested testing procedures were proposed to distinguish between the two stochastic and non-stochastic seasonal processes. When the two specifications are not based on strong theoretical foundations, both may be nested within a more general framework and standard (asymptotic) $F$ tests may be used to test the appropriate restrictions. Insignificant test statistics lead to non-rejection of the respective null hypotheses, whereas significant test statistics for both null hypotheses leads to rejection of both in favour of the more general model. In the non-nested case, where differences in theoretical approaches, in the auxiliary assumptions or in the approaches to econometric modelling predominate, a more general model is not entertained in the testing process. Therefore, rejection of the two non-nested models indicates that both are misspecified in ways not detected by standard (and possibly less powerful) diagnostic checks. However, there is no indication as to how to correct or accommodate the apparent misspecifications.

The procedures seem to work well in practice. Six empirical examples, using quarterly real macroeconomic time series for the United Kingdom highlighted various interpretations. In the case of Imports, the I model with a structural break was preferred to the PI model using the non-nested procedure, whereas the nested tests rejected both in favour of the general model. For Nondurables, both the PI and I models were rejected in favour of the general specification incorporating both PI and a structural break, using the nested and non-nested procedures. Thus, in the case of several variables, time series models which had passed standard diagnostic checks were rejected on the basis of evidence provided by a non-nested alternative or by a more general model not implied by the diagnostics.

Extensions of the tests proposed in this paper may take various forms. It was assumed throughout the analysis of the integrated model with a structural break that the value of $\tau$, the breakpoint, can be determined using a test for parameter constancy. Preferably, a joint test of $\tau$ and the non-nested alternative 
of PI simultaneously would obviate the need to be concerned with the sequential testing problem, and would also extend the applicability of the procedure (see Bera et al. (1992) for the derivation of a joint test of non-nested models together with departures from the auxiliary assumptions regarding the errors of a linear regression model). Extensions to multiple structural breaks, slowly changing breaks and continuous breaks could also be examined within the context of the problem analysed here. In each case, the PI model may be non-nested with respect to its I counterpart with known or unknown structural breaks.

\section{Acknowledgements}

The authors wish to thank three anonymous referees, John Geweke, Andrew Harvey, Marius Ooms, Adrian Pagan, Jeremy Smith, Ken Wallis and seminar participants at the London School of Economics, Monash University, National University of Singapore, and the Universities of Cambridge, Edinburgh, Glasgow, Tasmania and Warwick for helpful comments. The first author thanks the Royal Netherlands Academy of Arts and Sciences for its financial support. The second author wishes to acknowledge the financial support of the Australian Research Council and the Royal Netherlands Academy of Arts and Sciences. Much of this paper was written while the second author was visiting CentER at Tilburg University and the Tinbergen Institute at Erasmus University Rotterdam. An earlier version of the paper was presented at the Australasian meeting of the Econometric Society, Armidale, Australia, July 1994.

\section{References}

Andrews, D.W.K. (1993), Tests for parameter instability and structural change with unknown change point, Econometrica, 61, 821-856.

Bera, A.K., M.McAleer, M.H. Pesaran and M.J. Yoon (1992), Joint tests of nonnested models and general error specifications, Econometric Reviews, 11, 97-117.

Boswijk, H.P. and P.H. Franses (1996), Unit roots in periodic autoregressions, Journal of Time Series Analysis, 17, 221-245.

Boswijk, H.P., P.H. Franses and N. Haldrup (1996), Multiple unit roots in periodic autoregression, Journal of Econometrics, to appear.

Canova, F. and B.E. Hansen (1995), Are seasonal patterns constant over time? A test for seasonal stability, Journal of Business and Economic Statistics, 13, $237-252$

Dastoor, N.K. (1983), Some aspects of testing non-nested hypotheses, Journal of Econometrics, 21, 213-28. 
Dastoor, N.K. and M. McAleer(1989), Some power comparisons of joint and paired tests for nonnested models under local hypotheses, Econometric Theory, 5, 83-94.-94.

Davidson, R. and J.G. MacKinnon (1981), Several tests for model specification in the presence of alternative hypotheses, Econometrica, 49, 781-93.

Deaton, A.S. (1982), Model selection procedures, or, does the consumption function exist?, in G.C. Chow and P.Corsi (eds.), Evaluating the reliability of macro-economic models, New York: Wiley, 43-65.

Fisher, G.R. and M. McAleer (1981), Alternative procedures and associated tests of significance for non-nested hypotheses, Journal of Econometrics, 16, 103-19.

Franses, P.H. (1994), A multivariate approach to modelling univariate seasonal time series, Journal of Econometrics, 63, 133-151.

Franses, P.H. (1996a), Recent advances in modelling seasonality, Journal of Economic Surveys, 10, 299-345.

Franses, P.H. (1996b), Periodicity and stochastic trends in economic time series, Oxford: Oxford University Press.

Franses, P.H. and R. Paap (1994), Model selection in periodic autoregressions, Oxford Bulletin of Economics and Statistics, 56, 421-439.

Gourieroux, C., A. Monfort and A. Trognon (1983), Testing nested or nonnested hypotheses, Journal of Econometrics, 21, 83-115.

Harvey, A.C. and P.H.J. Todd (1983), Forecasting economic time series with structural and Box-Jenkins models (with discussion), Journal of Business and Economic Statistics, 1, 299-315.

Hylleberg, S. (1994), Modelling seasonal variation, in C.P. Hargreaves (ed.), Nonstationary time series analysis and cointegration, Oxford: Oxford University Press.

Johansen, S. (1991), Estimation and hypothesis testing of cointegration vectors in Gaussian vector autoregressive models, Econometrica, 59, 1551-80.

McAleer, M. (1995), The significance of testing empirical non-nested models, Journal of Econometrics, 67, 149-171.

McAleer, M. and M.H. Pesaran (1986), Statistical inference in non-nested econometric models, Applied Mathematics and Computation, 20, 271-311. 
Mizon, G.E. and J.-F. Richard (1986), The encompassing principle and its application to non-nested hypotheses, Econometrica, 54, 657-78.

Osborn, D.R. (1988), Seasonality and habit persistence in a life-cycle model of consumption, Journal of Applied Econometrics, 3, 255-66.

Osborn, D.R. (1991), The implications of periodically varying coefficients for seasonal time series processes, Journal of Econometrics, 48, 373-84.

Pagano, M. (1978), On periodic and multiple autoregressions, Annals of Statistics, 6, 1310-17.

Perron, P. (1989), The great crash, the oil price shock, and the unit root hypothesis, Econometrica, 57, 1361-1401.

Pesaran, M.H. (1974), On the general problem of model selection, Review of Economic Studies, 41, 153-71.

Pesaran, M.H. and A.D. Hall (1988), Tests of non-nested linear regression models subject to linear restrictions, Economics Letters, 27, 341-48.

Pesaran, M.H., R.P. Smith and J.S. Yeo (1985), Testing for structural stability and predictive failure: a review, The Manchester School, 53, 280-295.

Prothero, D.L. and K.F. Wallis (1976), Modelling macroeconomic time series (with discussion), Journal of the Royal Statistical Society A, 139, 468- 500.

Roy, S.N. (1953), On a heuristic method of test construction and its use in multivariate analysis, Annals of Mathematical Statistics, 24, 220-38.

Tiao, G. and M.R. Grupe (1980), Hidden periodic autoregressive-moving average models in time series data, Biometrika, 67, 365-73.

Vecchia, A.V. and R. Ballerini (1991), Testing for periodic autocorrelations in seasonal time series data, Biometrika, 78, 53-63.

Received January, 1996; Revised December, 1996. 